\title{
Coupling Characteristics of Fused Optical Fiber Coupler Formed with Single-Mode Fiber and Photonic Crystal Fiber Having Air Hole Collapsed Taper
}

\author{
Hirohisa Yokota, Hirotomo Yashima, Yoh Imai, and Yutaka Sasaki \\ Graduate School of Science and Engineering, Ibaraki University, 4-12-1 Nakanarusawa, Hitachi, Ibaraki 316-8511, Japan \\ Correspondence should be addressed to Hirohisa Yokota; hirohisa.yokota.ope@vc.ibaraki.ac.jp
}

Received 31 August 2016; Accepted 31 October 2016

Academic Editor: Jung Y. Huang

Copyright @ 2016 Hirohisa Yokota et al. This is an open access article distributed under the Creative Commons Attribution License, which permits unrestricted use, distribution, and reproduction in any medium, provided the original work is properly cited.

\begin{abstract}
Fused coupler forming with a single-mode fiber (SMF) and a photonic crystal fiber (PCF) is one of the solutions for optical coupling from a light source to a PCF. In this paper, we presented coupling characteristics of a fused fiber coupler formed with an ordinary SMF and a PCF having air hole collapsed taper. A prototype of SMF-PCF coupler with air hole collapsed taper was fabricated using $\mathrm{CO}_{2}$ laser irradiation. The coupling efficiency from SMF to PCF was $-6.2 \mathrm{~dB}$ at $1554 \mathrm{~nm}$ wavelength in the fabricated coupler. The structure of the SMF-PCF coupler to obtain high coupling efficiency was theoretically clarified by beam propagation analysis using an equivalent model of the coupler with simplification. It was clarified that appropriately choosing the prestretched or etched SMF diameter and the length of air hole collapsed region was effective to obtain high coupling efficiency that was a result of high extinction ratio at cross port and low excess loss. We also demonstrated that the diameter of prestretched SMF to obtain high coupling efficiency was insensitive to the air hole diameter ratio to pitch of the PCF in the air hole collapsed SMF-PCF coupler.
\end{abstract}

\section{Introduction}

A photonic crystal fiber (PCF) that has air holes around the center of its cross-section exhibits various interesting characteristics not seen in conventional optical fibers, such as an endlessly single mode, an extremely high/low nonlinearity, a flexible dispersion controllability, and a high birefringence [1]. Because of those unique properties, PCFs have been applied to functional fibers especially for dispersion compensations [2,3] and nonlinear fiber optics [4-7]. Recently, applications of PCFs as transmission lines also have been expected featuring PCF properties such as an endlessly single mode and a flexible dispersion controllability since low loss PCFs had been developed [8-10].

In a PCF application, an optical coupling from a light source to the PCF is important. Using a lens is the simplest solution for the optical coupling. However, a precise alignment of the light source, the lens, and the PCF facet is required. Using an ordinary single-mode fiber (SMF) is another solution for the optical coupling to the PCF, where the SMF is spliced to the PCF and the light is coupled to the PCF through the SMF. The advantages of the optical coupling using the SMF are an easy alignment and long time stability compared with that with the lens. An arc fusion splice or a butt joint with V-groove is usually used for splices of SMFs and PCFs. In the fiber splices, a mode field diameter mismatch and a Fresnel reflection at the boundary of the claddings cause splice losses. It had been reported that controlling air hole diameter is effective to reduce splice loss [11-13]. A damage of facet by high optical intensity is another problem in the fiber splice, especially in the splice of an SMF to a functional PCF using high nonlinearity such as a PCF for supercontinuum (SC) generation.

One of the solutions is a fused coupler forming with an SMF and a PCF. In [14], an optical coupling in the SMF-PCF coupler was reported, where the air holes were remaining in the coupler taper. However, a fiber coupler formed with PCF is essentially lossy even though the air holes are not collapsed since a fundamental mode cutoff is caused in a PCF taper [15]. Moreover, the prestretched SMF diameter should be carefully chosen to match effective indices of both fibers before coupler fabrication because the effective index of the 


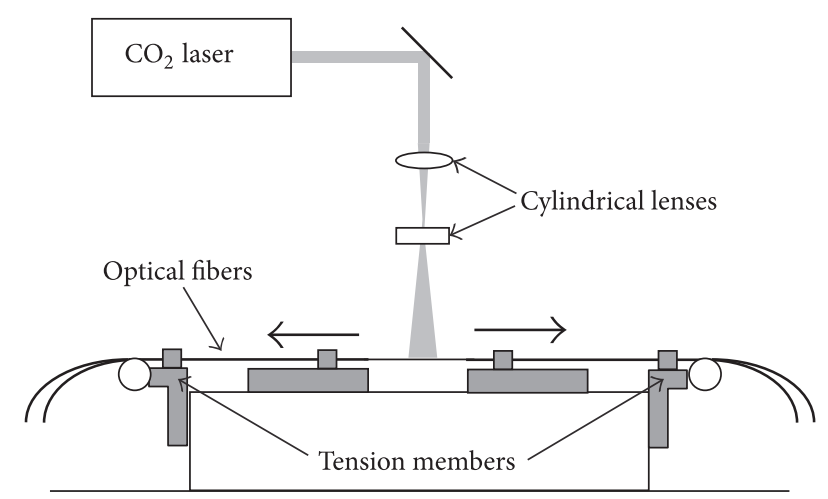

FIGURE 1: Setup for coupler fabrication using $\mathrm{CO}_{2}$ laser irradiation.

air hole remaining PCF strongly depends on PCF parameters such as an air hole diameter and an air hole pitch. Hence, an SMF-PCF coupler with air hole collapsed taper is worth investigating for a connection method of an SMF and a PCF. It is expected that the prestretched SMF diameter to obtain high coupling efficiency is insensitive to the PCF parameters since the air holes are collapsed in coupler taper.

In this paper, we investigate coupling characteristics of SMF-PCF couplers with air hole collapsed tapers. A prototype of air hole collapsed SMF-PCF coupler is fabricated using $\mathrm{CO}_{2}$ laser irradiation technique, and an optical coupling from SMF to PCF is confirmed. The structure of SMF-PCF coupler to obtain high coupling efficiency from the SMF to the PCF is theoretically clarified. We also demonstrate that the diameter of prestretched SMF to obtain high coupling efficiency is insensitive to the ratio of air hole diameter to pitch of the PCF in the air hole collapsed SMF-PCF coupler and clarify the wavelength characteristics of the coupler. The possibility of etching for SMF diameter reduction as an alternative technique of prestretch is examined.

\section{Fabrication of SMF-PCF Coupler}

We fabricated SMF-PCF couplers with air hole collapsed tapers using $\mathrm{CO}_{2}$ laser irradiation technique. Since this technique can precisely control the energy to heat fiber, it is suitable for PCF device fabrications in which the states of air holes are controlled $[16,17]$.

The coupler fabrication setup is shown in Figure 1. A pulsed $\mathrm{CO}_{2}$ laser with $14.285 \mathrm{kHz}$ repetition frequency was used for SMF-PCF coupler fabrications. The $\mathrm{CO}_{2}$ laser beam was irradiated to fibers through two cylindrical lenses to focus the beam in radial direction to fibers and to defocus the beam in longitudinal direction to fibers. The diameter of the beam on fibers was $\sim 1 \mathrm{~mm}$ in radial direction to fibers and was $\sim 14 \mathrm{~mm}$ in longitudinal direction to fibers. We used an ordinary telecommunication SMF (Corning SMF-28e) and a PCF with 6 air hole rings. A photograph of the PCF crosssection is shown in Figure 2. The pitch of air holes was $\sim 5.0 \mu \mathrm{m}$ and the diameter of air holes was $\sim 3.0 \mu \mathrm{m}$ except for ten large air holes with $\sim 4.8 \mu \mathrm{m}$ diameter surrounding the core and two small air holes elliptically. The cladding diameter of the PCF was $\sim 123 \mu \mathrm{m}$. In the experiment, the

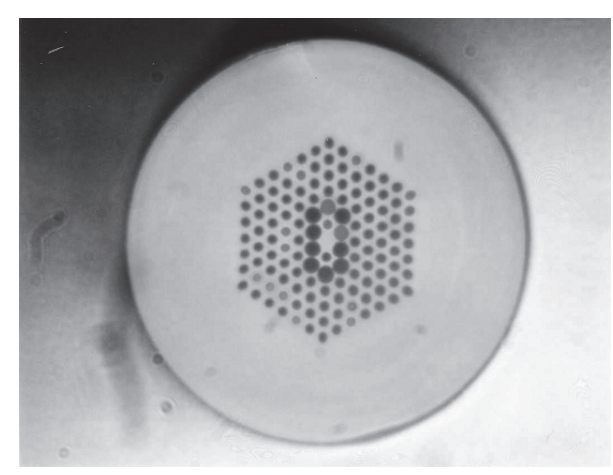

FIGURE 2: Cross-section of PCF for coupler fabrication.

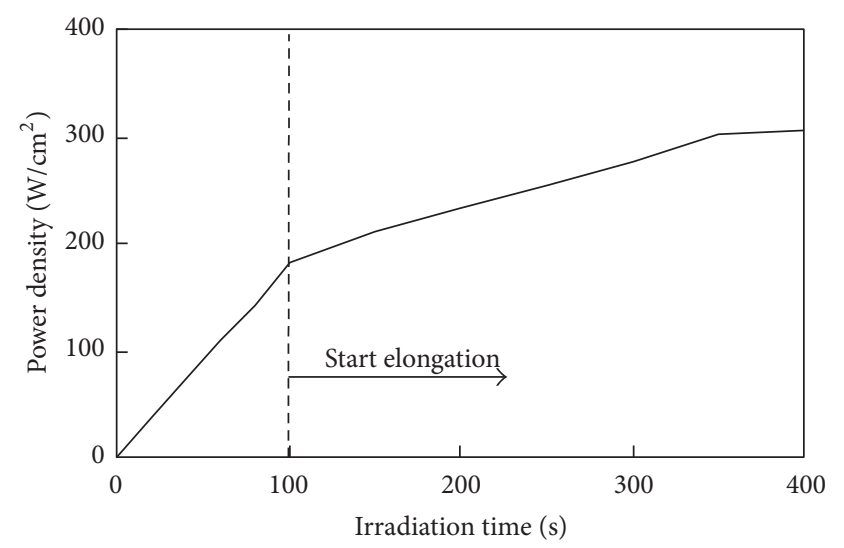

FIGURE 3: Irradiated laser power density in SMF-PCF coupler fabrication.

prestretch of the SMF to match effective indices was not performed because we would like to confirm the possibility of optical coupling from the SMF to the PCF.

In the SMF-PCF coupler fabrications, the $\mathrm{CO}_{2}$ laser beam was irradiated for 100 seconds to fuse the SMF and the PCF before elongation. After the fiber fusion, the fibers were elongated for 300 seconds with $60 \mu \mathrm{m} / \mathrm{s}$ elongation speed while the $\mathrm{CO}_{2}$ laser beam was irradiated. Since the temperature of $\mathrm{CO}_{2}$ laser irradiated fiber is decided by the laser power density and the fiber diameter, the irradiated laser power density was temporally changed as shown in Figure 3 to make the fiber temperature constant so that the air holes were collapsed and that the fiber deformation due to low viscosity could be avoided.

A prototype of air hole collapsed SMF-PCF coupler could be fabricated. Figure 4 shows photographs of the fabricated SMF-PCF coupler taper side view. It is found that the air holes of the PCF are collapsed in the taper transition region. The taper length of the SMF-PCF coupler defined as the distance between the points of $10 \%$ diameter reduction was $\sim 18 \mathrm{~mm}$, and the length of the taper waist was $\sim 5.5 \mathrm{~mm}$. Here, the length of the taper waist is defined as the distance between the points of $10 \%$ diameter increment from the minimum diameter. The minimum fiber diameter at the taper waist was $\sim 0.09$ times of the fiber diameter before elongation. The diameter of the SMF was slightly smaller than that of the PCF 


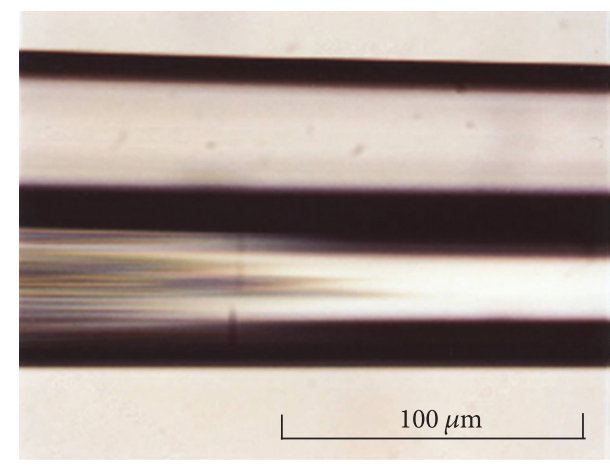

(a) Taper transition region

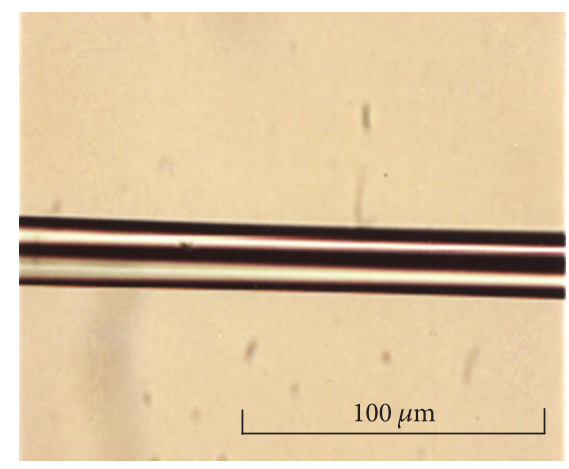

(b) Taper waist

FIgURE 4: Photographs of the fabricated SMF-PCF coupler taper side view.

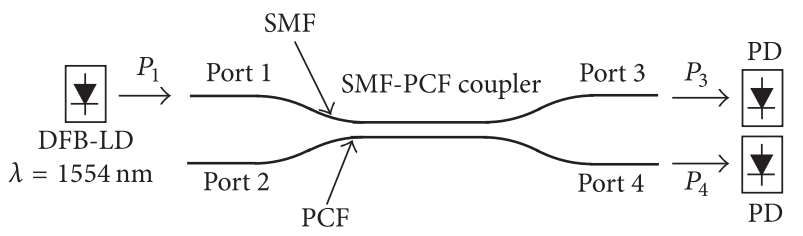

Figure 5: Setup for optical characteristics measurement.

at the taper waist. The length of the air hole collapsed region was $\sim 12.8 \mathrm{~mm}$.

We measured the coupling characteristics using the setup shown in Figure 5. The light from the laser diode was launched into port $1(\mathrm{SMF})$. The extinction ratios at port 3 (straight) and port 4 (cross) were estimated as $100 \times P_{3} /\left(P_{3}+\right.$ $\left.P_{4}\right)[\%]$ and $100 \times P_{4} /\left(P_{3}+P_{4}\right)$ [\%], respectively. Here, $P_{3}$ and $P_{4}$ are the output powers at port 3 and port 4 . The extinction ratio at port 3 was $11 \%$, and that at port 4 was $89 \%$. It was confirmed that optical coupling from SMF to PCF was obtained. The excess loss defined as $-10 \log \left[\left(P_{3}+P_{4}\right) / P_{1}\right][\mathrm{dB}]$ was $5.7 \mathrm{~dB}$, where $P_{1}$ is the input power. Considering the excess loss, the coupling efficiency from SMF to PCF was $-6.2 \mathrm{~dB}$.

Since the air holes are collapsed in the middle of coupler taper, the guided light can be easily lost in the case of contact with another material of compatible or higher refractive index. Hence, the SMF-PCF coupler should be packaged to avoid the contact of the coupler taper with another material in the use of the coupler. One of the solutions is covering the coupler taper with a hollow tube, where both ends of tube are sealed.

The coupling efficiency obtained in the experiment $(-6.2 \mathrm{~dB})$ is not sufficient in practical use. In the next section, we theoretically investigate the coupling characteristics of SMF-PCF couplers to clarify the optimum coupler structure for high coupling efficiency, that is, high extinction ratio at cross port and low excess loss.

\section{Theoretical Analysis of SMF-PCF Coupler}

Optical characteristics of SMF-PCF coupler can be obtained by calculating a light propagation in SMF-PCF coupler. The

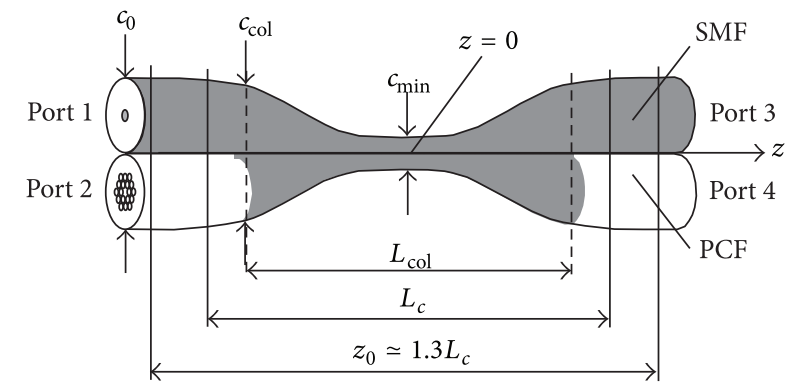

FIGURE 6: Analysis model of SMF-PCF coupler.

analysis model of the SMF-PCF coupler is shown in Figure 6. The shaded region of the PCF indicates that air holes are collapsed. The incident light is launched into port 1 . We assumed that the taper profile along $z$ could be expressed by the following function for the range of $z$ as $-L_{c} / 2 \leq z \leq L_{c} / 2$ ( $L_{c}$ : length of the tapered region):

$$
c(z)=c_{\min }+\left(c_{0}-c_{\min }\right)\left[\frac{2 z}{z_{0}}-\frac{1}{2 \pi} \sin \left(\frac{4 \pi z}{z_{0}}\right)\right]^{2},
$$

where $c(z)$ is the major diameter of the coupler at axial position $z, c_{0}$ is the initial diameter of coupler, and $c_{\min }$ is the minimum diameter at the center of coupler taper $(z=0) . z_{0}$ is the scalar length of the taper. As mentioned in the previous section, the length of the tapered region $L_{c}$ is defined as the distance between the points of $10 \%$ diameter reduction. It had been reported that the taper profile of the fused coupler using $\mathrm{CO}_{2}$ laser irradiation technique could be approximated by (1) [18]. In the taper profile, the relation between $z_{0}$ and $L_{c}$ is $z_{0} \simeq 1.3 L_{c} . L_{\text {col }}$ is the length of air hole collapsed region in coupler taper.

In the tapered region of the coupler, the air hole remaining PCF cross-section was replaced to the step index fiber cross-section with hexagonal inner cladding as shown in Figure 7(a), where it was assumed that the air holes in PCF cross-section had the same diameter for simplicity. We think that this assumption is roughly valid because the incident light is launched into port 1 of the SMF, and the optical coupling between SMF and PCF mainly occurs in 


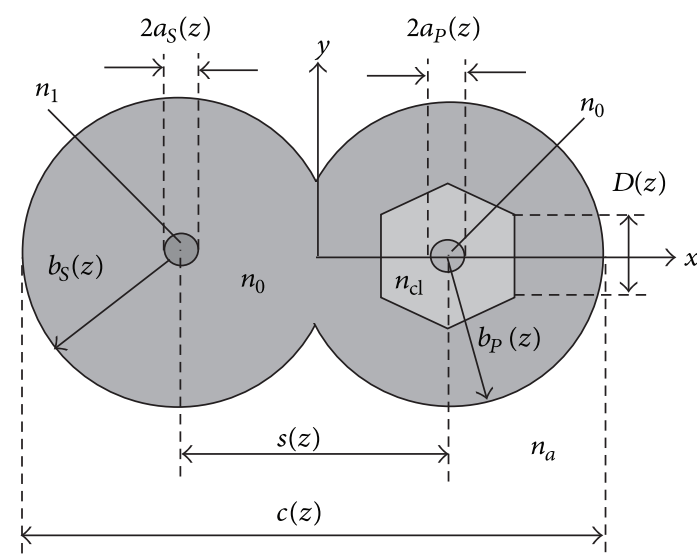

(a) Air hole remaining region

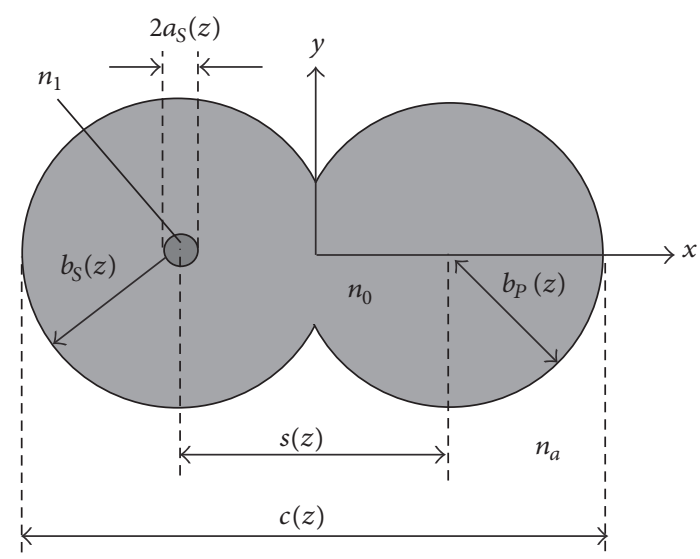

(b) Air hole collapsed region

FIGURE 7: Cross-section of SMF-PCF coupler taper.

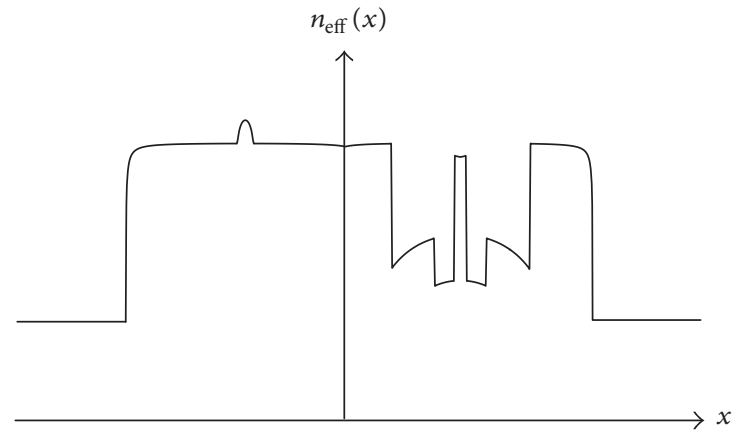

(a) Air hole remaining region

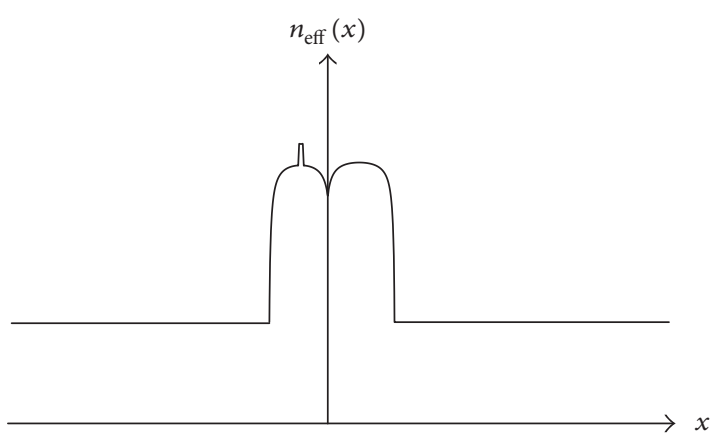

(b) Air hole collapsed region

FIGURE 8: Examples of effective index profiles in the cross-section of coupler taper.

the air hole collapsed region; hence it is considered that the existence of large air holes does not much affect the coupling characteristic of air hole collapsed coupler. The refractive index of the inner cladding is given as $n_{\mathrm{cl}}=\beta_{\mathrm{FSM}} / k$, where $\beta_{\mathrm{FSM}}$ is a propagation constant of fundamental spacefilling mode and $k$ is a wave number in vacuum [19]. The propagation constant $\beta_{\mathrm{FSM}}$ at the axial position $z$ can be calculated using the air hole pitch $\Lambda(z)$, the air hole diameter $d(z)$, the wave number $k$, and the indices of materials (pure silica and air). The air hole pitch $\Lambda(z)$ was assumed to be $\Lambda(z)=\left[c(z) / c_{0}\right] \Lambda_{0}$, where $\Lambda_{0}$ is the original air hole pitch before elongation. The air hole diameter $d(z)$ was assumed as

$$
d(z)= \begin{cases}\frac{c(z)}{c_{0}} d_{0} \times \frac{c(z)-c_{\mathrm{col}}}{c_{0}-c_{\mathrm{col}}}, & c(z)>c_{\mathrm{col}} \\ 0, & c(z) \leq c_{\mathrm{col}},\end{cases}
$$

where $c_{\mathrm{col}}$ is the major diameter of the coupler defined as air holes which are collapsed for $c(z) \leq c_{\text {col }}$. A side length of the hexagonal inner cladding is given as $D(z)=(2 N+1) \Lambda(z) / 2$, where $N$ is the number of air hole rings. The radius of the PCF core with refractive index $n_{0}$ at $z$ is defined as $a_{P}(z)=$ $\Lambda(z) / \sqrt{3}$ [20]. The air hole collapsed PCF cross-section was treated as the cross-section of pure silica rod as shown in Figure $7(b)$. The radius of the SMF core with refractive index $n_{1}$ at $z$ is given as $a_{S}(z)=\left[c(z) / c_{0}\right] a_{S 0}$, where $a_{S 0}$ is the initial core radius of the SMF before elongation. The cladding radii of SMF and PCF are given as $b_{S}(z)=\left[c(z) / c_{0}\right] b_{S 0}$ and $b_{P}(z)=\left[c(z) / c_{0}\right] b_{P 0}$ using the initial cladding radii $b_{S 0}$ and $b_{P 0}$, respectively. $s(z)$ is defined as the length between the centers of SMF and PCF cross-sections at $z$.

In the light propagation analysis, we replaced the SMFPCF coupler to the equivalent slab waveguide coupler using effective index method [21]. Examples of effective index profiles in the cross-section of coupler taper are shown in Figure 8 . The light propagation in the equivalent slab waveguide coupler was calculated using the finite difference beam propagation method (FD-BPM) [22-24]. We can obtain the extinction ratios at the output ports, the excess loss, and the coupling efficiency from port 1 to port 4 using the calculated field distribution at the end of SMF-PCF coupler taper. As described in the previous section, the extinction ratios at port 3 and port 4 are defined as $100 \times P_{3} /\left(P_{3}+P_{4}\right)$ [\%] and $100 \times$ $P_{4} /\left(P_{3}+P_{4}\right)$ [\%], where $P_{3}$ and $P_{4}$ are output powers at ports 3 and 4 . They are calculated by the overlap integrals of the output field distribution and the normal modes at ports 3 and 4. The excess loss is given as $-10 \log \left[\left(P_{3}+P_{4}\right) / P_{1}\right][\mathrm{dB}]$, where $P_{1}$ is the input optical power at port 1 . The coupling efficiency from port 1 to port 4 is defined as $10 \log \left(P_{4} / P_{1}\right)[\mathrm{dB}]$. 


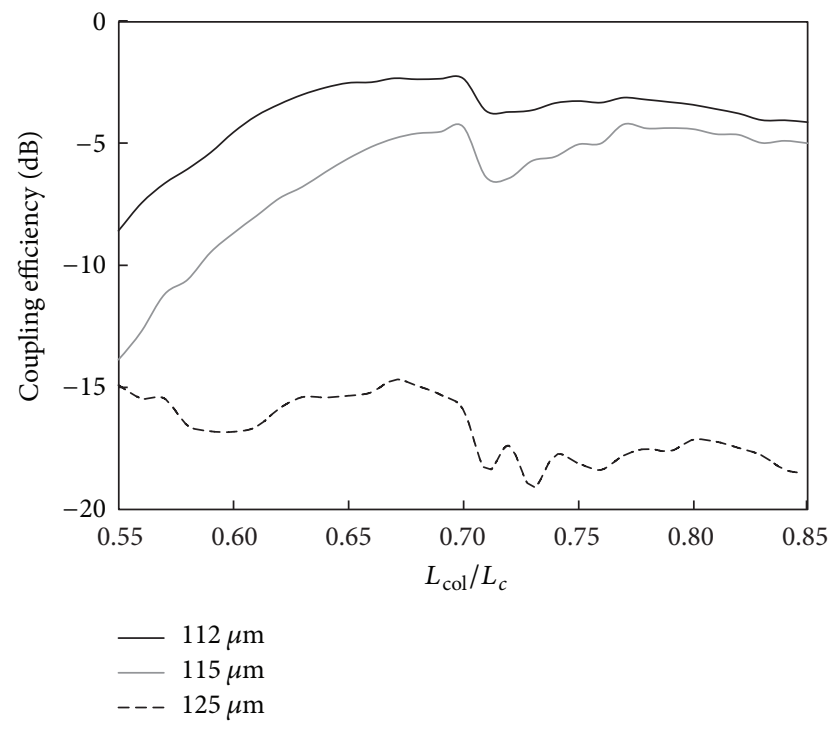

Figure 9: Coupling efficiencies for the length of the air hole collapsed region with various SMF diameters with prestretching.

The parameters for numerical calculation are listed as follows. The operating wavelength was selected as $1550 \mathrm{~nm}$. The refractive index of pure silica, that is, the material of the SMF cladding, the PCF core, and the PCF outer cladding, was chosen as $n_{0}=1.4443$ considering the operating wavelength. The refractive index of the SMF core was $n_{1}=1.4487$. The outer medium is air; hence the refractive index of the outer medium is $n_{a}=1$. The initial cladding diameter of the SMF was chosen as $2 b_{S 0}=112 \sim 125 \mu \mathrm{m}$ considering prestretch before coupler fabrication to match the effective indices (the propagation constants) of SMF and PCF in the coupler taper. The initial core diameter of the SMF was $2 a_{S 0}=$ $8.2 \mu \mathrm{m} \times 2 b_{S 0} / 125$ assuming to use an ordinary single-mode telecommunication fiber, where the unit of $2 b_{S 0}$ is $\mu \mathrm{m}$. The initial cladding diameter of the PCF with 6 air hole rings was $125 \mu \mathrm{m}$. The initial air hole diameter and pitch of the PCF were $3 \mu \mathrm{m}$ and $5 \mu \mathrm{m}$, respectively (i.e., the ratio of air hole diameter to pitch $d / \Lambda=0.6)$. The length of the tapered region of the SMF-PCF coupler was $20 \mathrm{~mm}$.

Figure 9 shows coupling efficiencies for the length of the air hole collapsed region $L_{\text {col }}$ (shown in Figure 6) with various SMF diameters. The degree of fusion defined as $s(z) /\left(b_{S}(z)+\right.$ $\left.b_{P}(z)\right)$ and the elongation ratio $c_{\min } / c_{0}$ were set as 0.85 and 0.1 , respectively. It is found that the coupling efficiency strongly depends on SMF diameter. Maximum coupling efficiency was $-2.3 \mathrm{~dB}$ for $L_{\text {col }}=0.7 L_{c}$ with $112 \mu \mathrm{m}$ SMF diameter. Properly selecting prestretched SMF diameter reduces the asymmetry of effective indices between SMF and PCF in the coupler taper. That makes the extinction ratio at the cross port higher; hence large coupling efficiency can be obtained.

As shown in Figure 9, the coupling efficiency becomes lower as the length of the air hole collapsed region is shorter except for $125 \mu \mathrm{m}$ SMF diameter. It is also found that there is a dip around $L_{\text {col }} \sim 0.71 L_{c}$ in each relation of the coupling efficiency and the length of the air hole collapsed region. To discuss these results, we calculated the extinction ratio at the

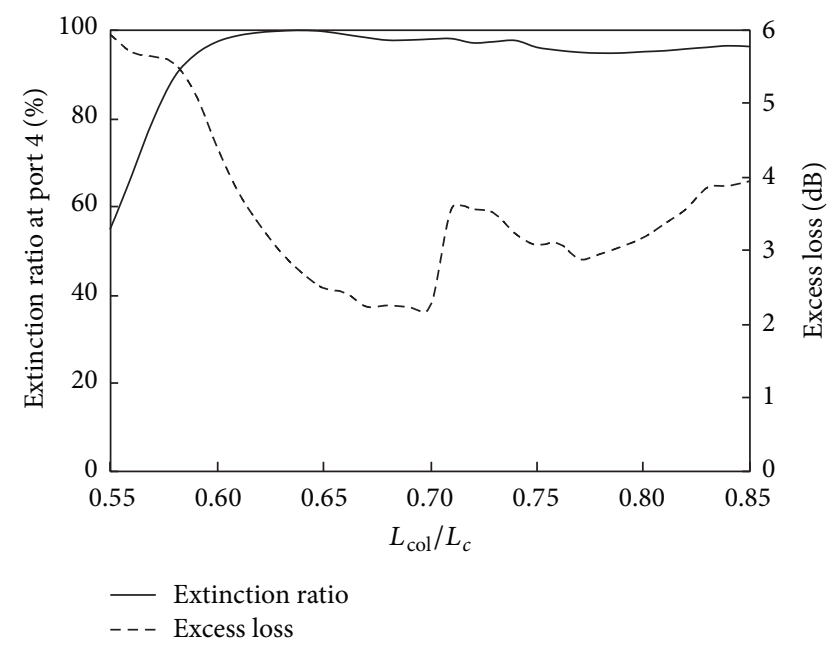

FIgURE 10: Extinction ratio at the cross port and excess loss for the length of the air hole collapsed region.

cross port (port 4) and the excess loss for the length of the air hole collapsed region. The results are shown in Figure 10, where the SMF diameter was $112 \mu \mathrm{m}$. As the length of the air hole collapsed region is shorter, the extinction ratio at cross port is lower and the excess loss is larger. It is considered that the low extinction ratio is induced by the effective index mismatch between the SMF and the air hole remaining PCF in the coupler taper. The large excess loss is induced by the fundamental mode cutoff [15] in the air hole remaining PCF taper. The reason of the coupling efficiency dip around $L_{\text {col }} \sim$ $0.71 L_{c}$ is the excess loss increment. It is considered that the excess loss increment is caused by the radiation at the taper transition region in which there is a boundary of "air hole remaining" and "air hole collapsed".

To examine the origin of the excess loss, the evolution of the intensity distribution was plotted. Figure 11 shows the evolution of the intensity distribution. Parameters for numerical calculation are similar to those for calculation of the extinction ratio and the excess loss shown in Figure 10. It is found that radiations are generated after propagation through the tapered region. The radiations that are the origin of excess loss are generated in the modal conversions at the taper transition region of the side of output ports. In the coupling region, there is a mode displacement along the $x$ axis. The displacement can be explained as an intermodal interference in the coupling region. Since the diameters of fibers are decreased by elongation and the air holes of the PCF are collapsed in coupler taper, the coupling region operates as a multimode waveguide with an air cladding. In the taper transition region of the side of input ports, the incident core mode of SMF is converted to a cladding mode that excites more than one guided mode of the coupling region. In the taper transition region of the side of output ports, the guided modes are converted to the cladding modes of SMF and PCF, and they are converted to the core modes. In the modal conversion from the cladding modes to the core mode, the mismatch components are converted to radiation modes. It is considered that properly designing the taper profile and the 


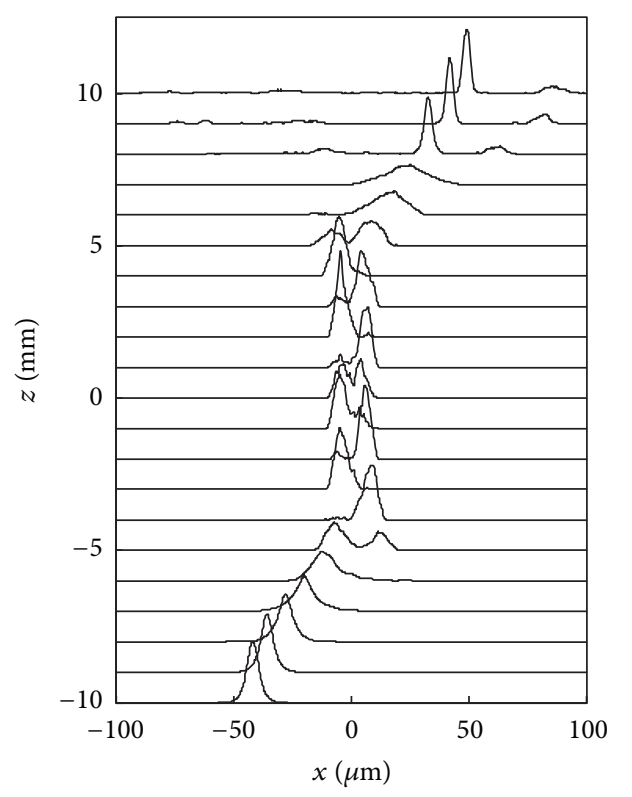

FIGURE 11: Evolution of intensity distribution in SMF-PCF coupler.

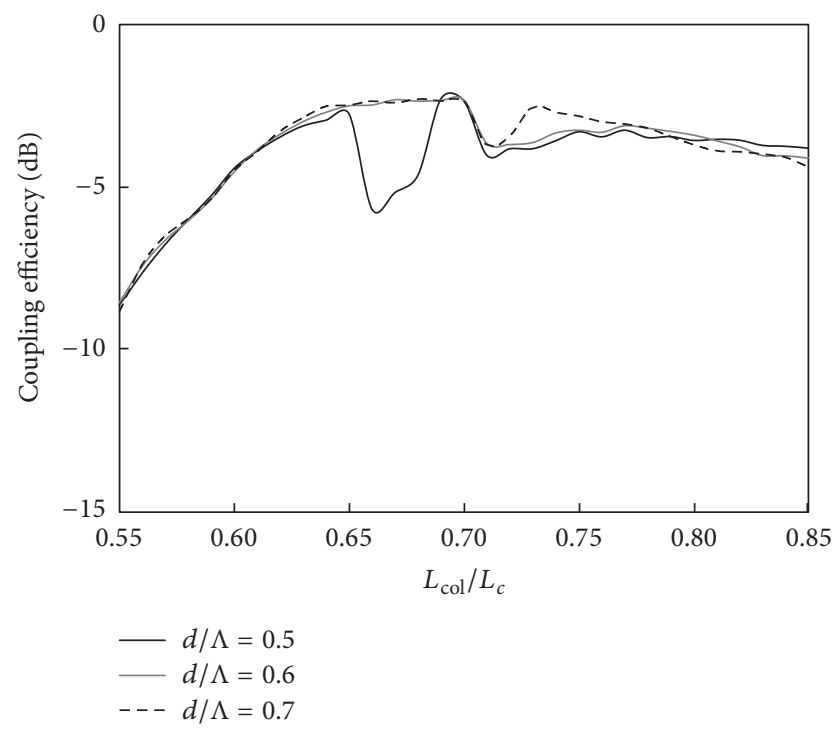

FIGURE 12: Coupling efficiencies for the length of the air hole collapsed region with various air hole ratios.

length of the air hole collapsed region is effective to reduce the radiations caused by nonadiabatic modal conversions.

The dependence of air hole diameter ratio to pitch of the PCF for coupling characteristics was also examined. Figure 12 shows coupling efficiencies for the length of the air hole collapsed region $L_{\text {col }}$ with various air hole ratios, where the SMF diameter, the degree of fusion, and the elongation ratio were $112 \mu \mathrm{m}, 0.85$, and 0.1 , respectively. It is found that $\sim-2.3 \mathrm{~dB}$ of coupling efficiency can be obtained for each air hole ratio. Hence, it was clarified that the prestretched SMF diameter for high coupling efficiency was insensitive to the air hole ratio of the PCF in the SMF-PCF coupler with air hole collapsed taper. This is an advantage of the air hole collapsed

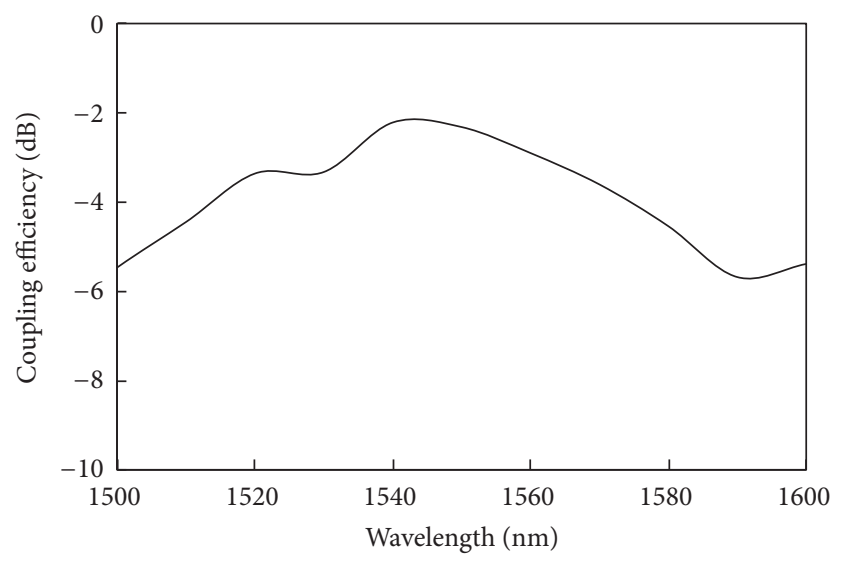

Figure 13: Wavelength characteristics of coupling efficiency.

SMF-PCF couplers compared with the SMF-PCF couplers with air hole remaining tapers because the prestretched SMF diameters should be carefully chosen for the air hole ratios in the air hole remaining coupler fabrications.

Wavelength characteristics of coupling efficiency were examined. The numerical result is shown in Figure 13, where the taper length, the SMF diameter, the degree of fusion, and the elongation ratio were $20 \mathrm{~mm}, 112 \mu \mathrm{m}, 0.85$, and 0.1 , respectively. The length of the air hole collapsed region was chosen as $0.7 L_{c}$. The FWHM of the coupling efficiency was $\sim 84 \mathrm{~nm}$. The reason of the coupling efficiency decrement apart from the wavelength of the efficiency peak was the decrement of the extinction ratio at cross port. It is caused by the wavelength dependence of the fiber effective indices.

Etching of SMF cladding is considered as another solution to reduce SMF diameter for propagation constant matching in coupler taper. The possibility of the etching was theoretically examined. The degree of fusion and the elongation ratio were chosen as 0.85 and 0.1 , respectively. The initial cladding diameter of the SMF was chosen as $2 b_{S 0}=112 \sim$ $125 \mu \mathrm{m}$ while the initial core diameter was $2 a_{S 0}=8.2 \mu \mathrm{m}$. The initial air hole diameter and pitch of the PCF were $3 \mu \mathrm{m}$ and $5 \mu \mathrm{m}$, respectively. Coupling efficiencies for the length of the air hole collapsed region $L_{\text {col }}$ are shown in Figure 14. It was confirmed that etching the SMF cladding was also effective for propagation constant matching. Maximum coupling efficiency was $-2.6 \mathrm{~dB}$ for $L_{\text {col }}=0.7 L_{c}$ with $112 \mu \mathrm{m}$ SMF diameter.

In the theoretical studies, we clarified that the coupling efficiency to the cross port in the air hole collapsed SMF-PCF coupler can be maximized by appropriately choosing the SMF diameter and the length of air hole collapsed region. In our numerical examples, up to $-2 \mathrm{~dB}$ coupling efficiency could be obtained.

\section{Conclusions}

In this paper, coupling characteristics of SMF-PCF couplers with air hole collapsed tapers were investigated. A prototype of the air hole collapsed SMF-PCF coupler was fabricated using $\mathrm{CO}_{2}$ laser irradiation. It was confirmed that the 


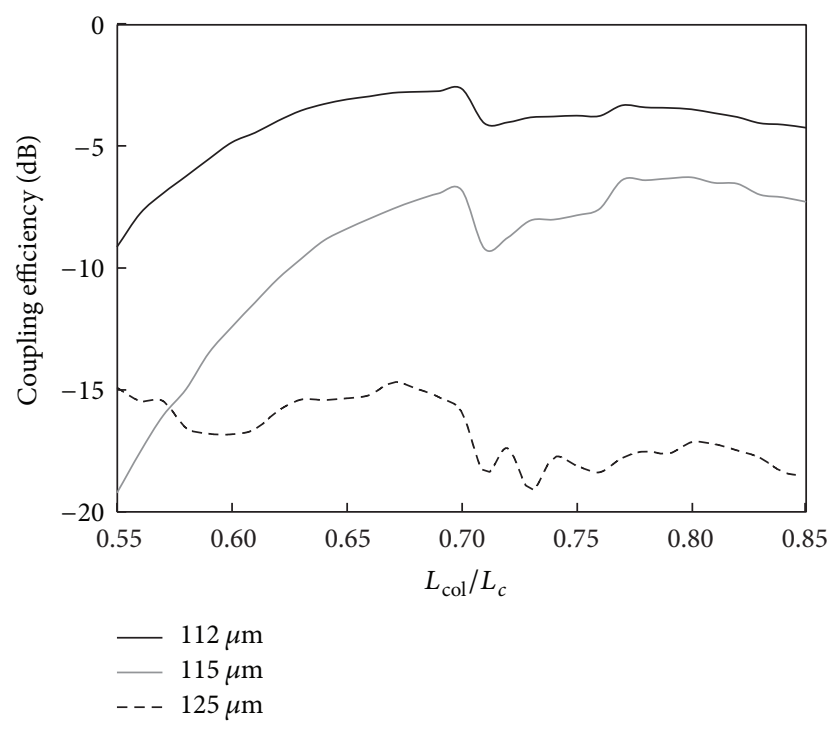

Figure 14: Coupling efficiencies for the length of the air hole collapsed region with various SMF diameters with etching.

coupling from the SMF to the PCF could be obtained. The coupling efficiency was $-6.2 \mathrm{~dB}$ at $1554 \mathrm{~nm}$ wavelength. Theoretical studies were performed to obtain high coupling efficiency in the air hole collapsed SMF-PCF coupler by beam propagation analysis using an equivalent model of the coupler with simplification. It was clarified that appropriately choosing the prestretched or etched SMF diameter and the length of air hole collapsed region was effective to obtain high coupling efficiency that was a result of high extinction ratio at cross port and low excess loss. In our numerical results, up to $-2 \mathrm{~dB}$ of coupling efficiency could be obtained. It was found that the SMF diameter for high coupling efficiency was insensitive to the ratio of air hole diameter to pitch of the PCF. It is an advantage of the air hole collapsed SMF-PCF coupler compared with the air hole remaining SMF-PCF coupler. Wavelength characteristics of the air hole collapsed SMFPCF coupler were examined. FWHM was $\sim 84 \mathrm{~nm}$ in our numerical result. Experimental verifications of the theoretical results are future issues.

\section{Competing Interests}

The authors declare that they have no competing interests.

\section{References}

[1] P. Russell, "Photonic crystal fibers," Science, vol. 299, no. 5605, pp. 358-362, 2003.

[2] T. A. Birks, D. Mogilevtsev, J. C. Knight, and P. S. J. Russell, "Dispersion compensation using single-material fibers," IEEE Photonics Technology Letters, vol. 11, no. 6, pp. 674-676, 1999.

[3] L. P. Shen, W.-P. Huang, G. X. Chen, and S. S. Jian, "Design and optimization of photonic crystal fibers for broad-band dispersion compensation," IEEE Photonics Technology Letters, vol. 15, no. 4, pp. 540-542, 2003.
[4] A. V. Husakou and J. Herrmann, "Supercontinuum generation, four-wave mixing, and fission of higher-order solitons in photonic-crystal fibers," Journal of the Optical Society of America $B$, vol. 19, no. 9, pp. 2171-2182, 2002.

[5] W. J. Wadsworth, N. Joly, J. C. Knight, T. A. Birks, F. Biancalana, and P. S. J. Russell, "Supercontinuum and four-wave mixing with Q-switched pulses in endlessly single-mode photonic crystal fibres," Optics Express, vol. 12, no. 2, pp. 299-309, 2004.

[6] K. K. Chow, C. Shu, C. Lin, and A. Bjarklev, "Polarizationinsensitive widely tunable wavelength converter based on fourwave mixing in a dispersion-flattened nonlinear photonic crystal fiber," IEEE Photonics Technology Letters, vol. 17, no. 3, pp. 624-626, 2005.

[7] P. A. Andersen, T. Tokle, Y. Geng, C. Peucheret, and P. Jeppesen, "Wavelength conversion of a 40-Gb/s RZ-DPSK signal using four-wave mixing in a dispersion-flattened highly nonlinear photonic crystal fiber," IEEE Photonics Technology Letters, vol. 17, no. 9, pp. 1908-1910, 2005.

[8] K. Tajima, J. Zhou, K. Nakajima, and K. Sato, "Ultralow loss and long length photonic crystal fiber," Journal of Lightwave Technology, vol. 22, no. 1, pp. 7-10, 2004.

[9] H. Kubota, S. Kawanishi, S. Koyanagi, M. Tanaka, and S. Yamaguchi, "Absolutely single polarization photonic crystal fiber," IEEE Photonics Technology Letters, vol. 16, no. 1, pp. 182184, 2004.

[10] K. Nakajima, J. Zhou, K. Tajima, K. Kurokawa, C. Fukai, and I. Sankawa, "Ultrawide-band single-mode transmission performance in a low-loss photonic crystal fiber," Journal of Lightwave Technology, vol. 23, no. 1, pp. 7-12, 2005.

[11] L. Xiao, M. S. Demokan, W. Jin, Y. Wang, and C.-L. Zhao, "Fusion splicing photonic crystal fibers and conventional single-mode fibers: microhole collapse effect," Journal of Lightwave Technology, vol. 25, no. 11, pp. 3563-3574, 2007.

[12] H. Hasegawa, M. Kikegawa, M. Yoshida, T. Hirooka, and M. Nakazawa, "Observation of optimum air-hole tapering of splicing between a conventional fiber and a photonic crystal fiber and analysis of reduction of Fresnel reflection," IEICE Transactions on Electronics (Japanese Edition), vol. J88, no. 10, pp. 779-787, 2005.

[13] H. Yokota, H. Yashima, Y. Imai, and Y. Sasaki, "Reduction of splice loss between single-mode optical fiber and high nonlinear photonic crystal fiber by air hole diameter control with $\mathrm{CO}_{2}$ laser irradiation," IEICE Transactions on Electronics (Japanese Edition), vol. 94, no. 2, pp. 53-56, 2011.

[14] J. Liu, T.-H. Cheng, Y.-K. Yeo et al., "Light beam coupling between standard single mode fibers and highly nonlinear photonic crystal fibers based on the fused biconical tapering technique," Optics Express, vol. 17, no. 5, pp. 3115-3123, 2009.

[15] H. C. Nguyen, B. T. Kuhlmey, M. J. Steel et al., "Leakage of the fundamental mode in photonic crystal fiber tapers," Optics Letters, vol. 30, no. 10, pp. 1123-1125, 2005.

[16] H. Yokota, H. Kawashiri, and Y. Sasaki, "Design and fabrications of photonic crystal fiber couplers with air hole controlled tapers," IEICE Transactions on Electronics, vol. 91, no. 7, pp. 1136$1141,2008$.

[17] H. Yokota, Y. Ito, H. Kawashiri et al., "Fabrication of polarization-maintaining photonic crystal fiber coupler with air hole state control using $\mathrm{CO}_{2}$ laser irradiation technique," IEICE Transactions on Electronics, vol. 95, no. 10, pp. 1689-1691, 2012.

[18] H. Yokota, T. Hasegawa, Y. Satoda, E. Sugai, and Y. Sasaki, "Characteristics of fused optical fiber grating couplers with tapered shapes," Optical Review, vol. 6, no. 3, pp. 173-179, 1999. 
[19] T. A. Birks, J. C. Knight, and P. St. J. Russell, "Endlessly singlemode photonic crystal fiber," Optics Letters, vol. 22, no. 13, pp. 961-963, 1997.

[20] M. Koshiba and K. Saitoh, "Applicability of classical optical fiber theories to holey fibers," Optics Letters, vol. 29, no. 15, pp. 17391741, 2004.

[21] K. Okamoto, "Theoretical investigation of light coupling phenomena in wavelength-flattened couplers," Journal of Lightwave Technology, vol. 8, no. 5, pp. 678-683, 1990.

[22] Y. Chung and N. Dagli, "Assessment of finite difference beam propagation method," IEEE Journal of Quantum Electronics, vol. 26, no. 8, pp. 1335-1339, 1990.

[23] R. Scarmozzino and R. M. Osgood, "Comparison of finitedifference and Fourier-transform solutions of the parabolic wave equation with emphasis on integrated-optics applications," Journal of the Optical Society of America A, vol. 8, no. 5, pp. 724-731, 1991.

[24] G. R. Hadley, "Transparent boundary condition for beam propagation," Optics Letters, vol. 16, no. 9, pp. 624-626, 1991. 


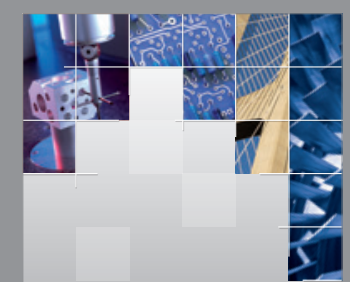

\section{Enfincering}
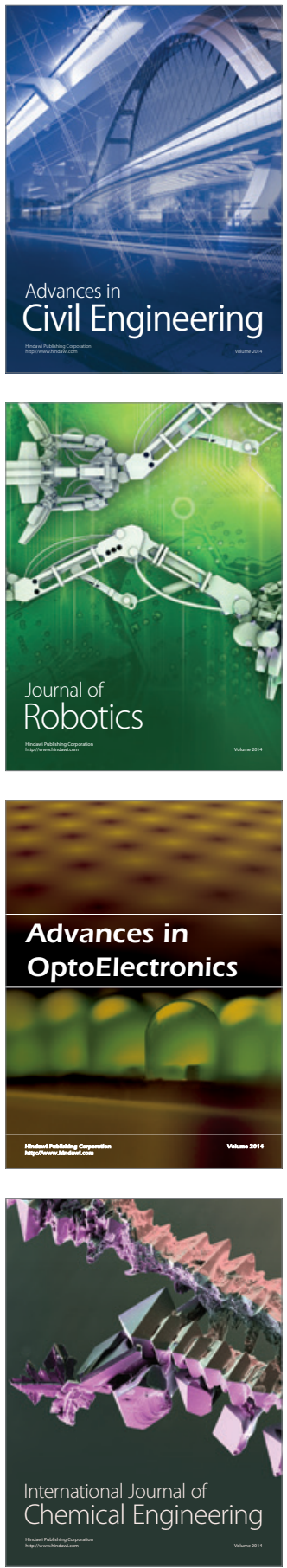

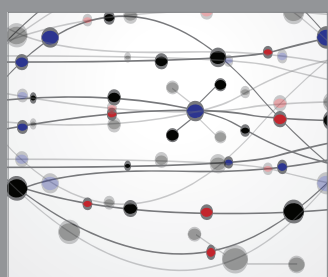

The Scientific World Journal

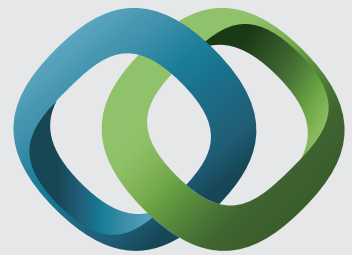

\section{Hindawi}

Submit your manuscripts at

http://www.hindawi.com
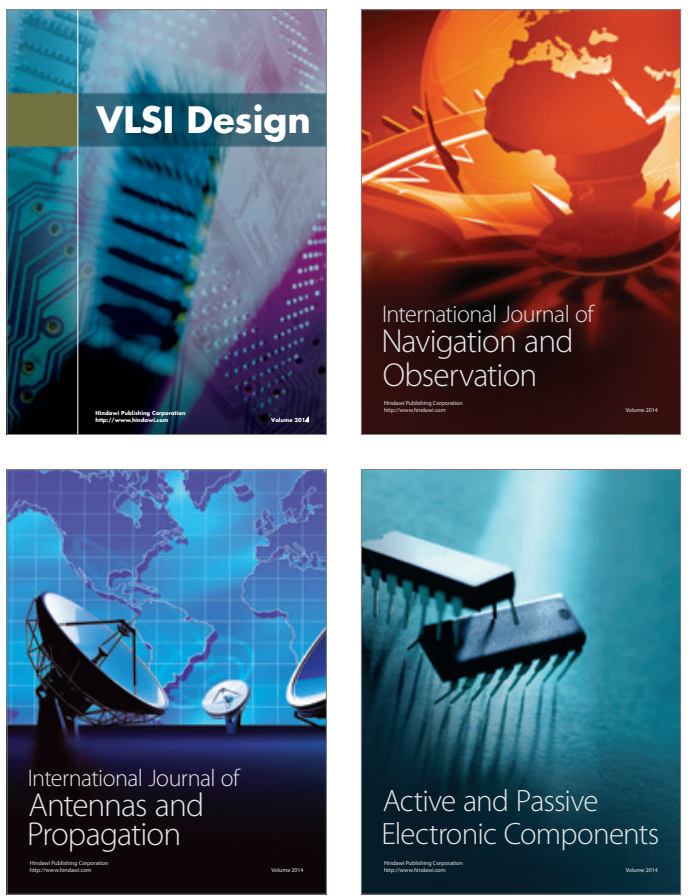
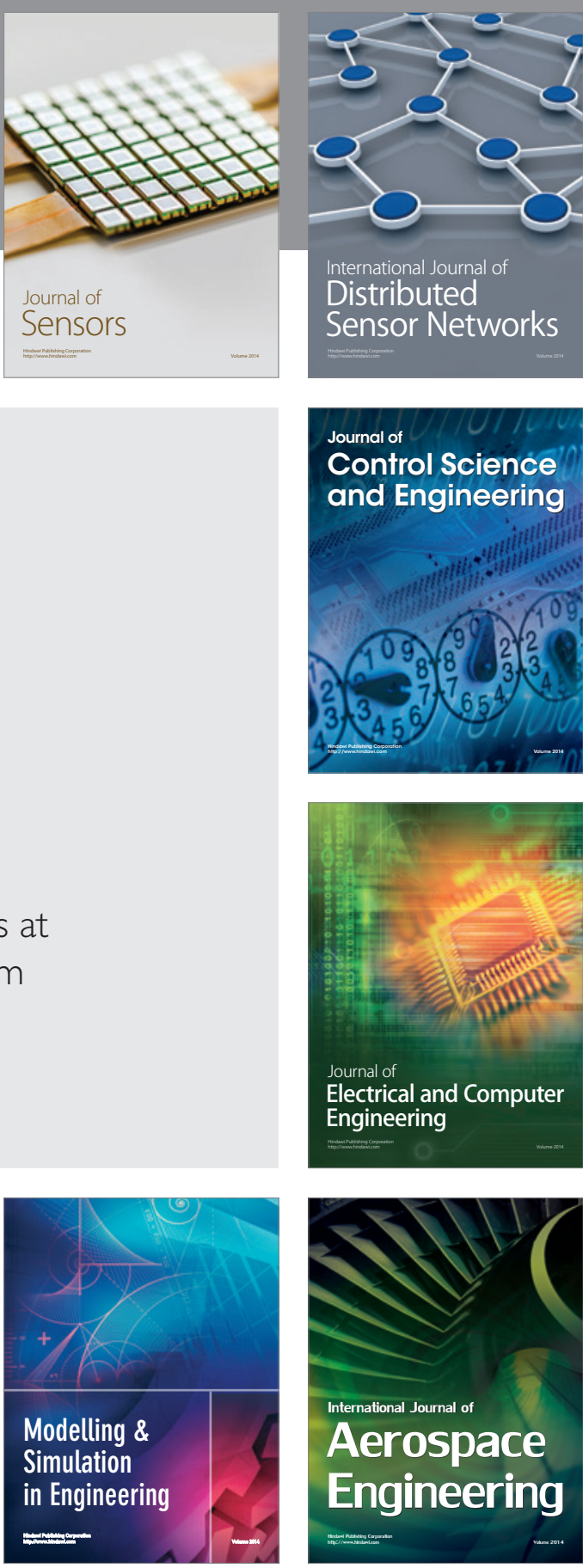

International Journal of

Distributed

Sensor Networks

Journal of

Control Science

and Engineering
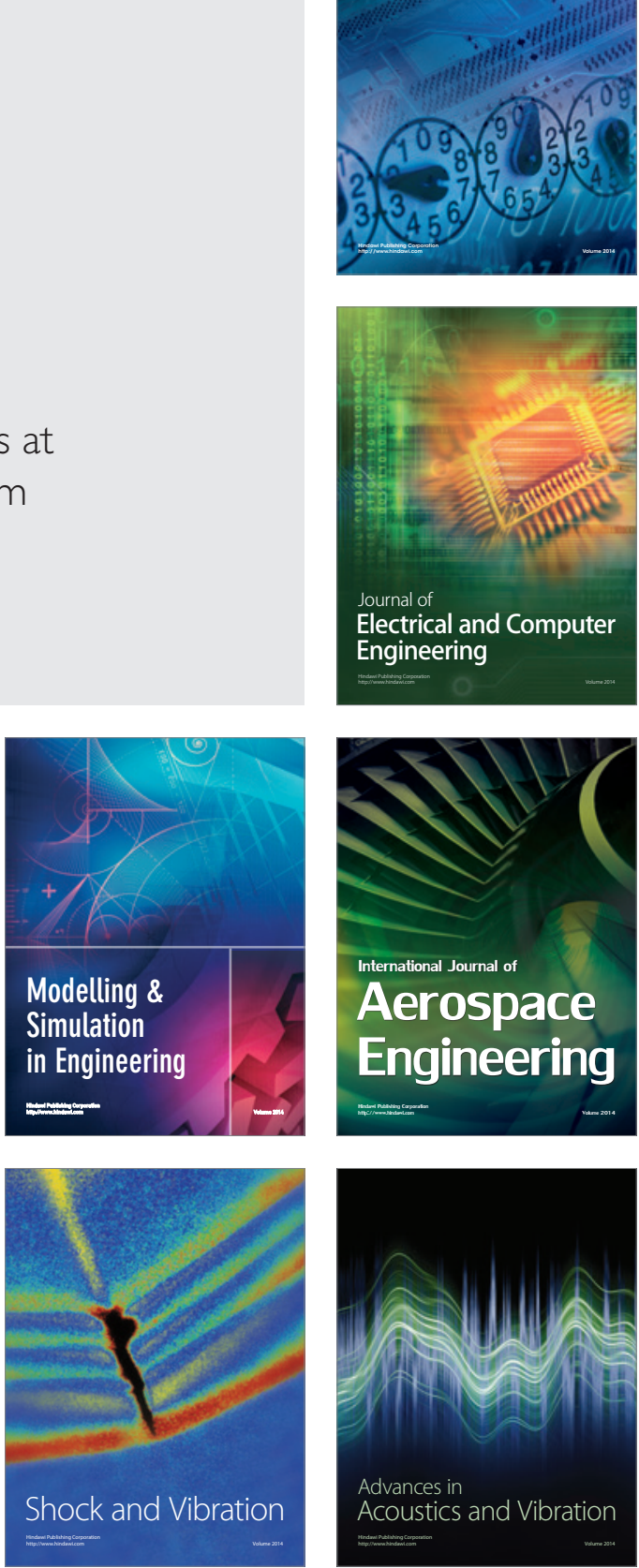